\title{
Multilaterale Entwicklungszusammenarbeit: Strukturwandel und Geberpräferenzen
}

\author{
Stephan Klingebiel
}

Zusammenfassung: Die Unterscheidung zwischen bi- und multilateraler EZ ist oftmals nicht eindeutig möglich, vielmehr gibt es verschiedene Zwischen- und Sonderformen. Grundsätzlich gibt es zwei Sichtweisen auf das multilaterale EZ-System: 1) Die Perspektive eines funktionsfähigen „Patchwork-Ansatzes“ für globale Herausforderungen. 2) Die Perspektive eines fragmentierten und wenig leistungsfähigen multilateralen Systems. Der vorliegende Beitrag analysiert daher strukturelle Veränderungen der multilateralen EZ und die Implikationen für die Qualität von EZ.

Schlüsselwörter: Entwicklungszusammenarbeit · Multilaterale Entwicklungszusammenarbeit · Wirksamkeit · Fragmentierung · Proliferation

\section{Multilateral Development Cooperation: Structural Change and Donor Preferences}

\begin{abstract}
It is rarely possible in practice to draw a clear distinction between bilateral development cooperation and multilateral development cooperation, as there are a number of overlaps and special forms. Essentially, there are two perspectives on multilateral development cooperation as a system: 1) It represents a functioning "patchwork" approach to global challenges. 2) It is strongly fragmented and inefficient. It is against this backdrop that this article analyses the structural changes taking place within multilateral development cooperation and their implications for the quality of development cooperation.
\end{abstract}

Keywords: Development cooperation - Multilateral development cooperation - Effectiveness · Fragmentation · Proliferation

\footnotetext{
Online publiziert: 20.11 .2013

Dr. S. Klingebiel $(\bowtie)$

Deutsches Institut für Entwicklungspolitik,

Tulpenfeld 6, 53113 Bonn, Deutschland

E-Mail: stephan.klingebiel@die-gdi.de
}

(C) Die Autor(en) 2013. Dieser Artikel ist auf Springerlink.com mit Open Access verfügbar. 


\section{Einleitung}

\subsection{Neue Relevanz des Themas?}

Entwicklungsforschung und Forschung über Entwicklungszusammenarbeit (EZ) befassen sich meist nur am Rande mit multilateraler EZ. Im Mittelpunkt stehen meist übergreifende Fragen - etwa nach dem Beitrag von EZ zu wirtschaftlichem Wachstum, nach den Einflüssen von EZ auf die Governance-Systeme in Partnerländern, nach der Wirksamkeit von Modalitäten und Instrumenten (Programmfinanzierung etc.) der EZ - oder einzelne Akteure wie die Weltbankgruppe und die Europäische Union.

In den vergangenen rund fünf Jahren sind jedoch verstärkt Fragen aufgegriffen worden, die sich direkt oder indirekt mit dem multilateralen EZ-System befassen. Zum Teil wurden diese Forschungsanstöße von der EZ-Praxis gegeben, zum Teil stammen sie aus der Entwicklungsforschung. Hier lassen sich vor allem zwei zum Teil überlappende Debatten unterscheiden. Erstens das Interesse von Geberländern an neuen evidenzbasierten Analysen zur Arbeit einzelner multilateraler Einrichtungen. Da rund die Hälfte aller Mitglieder des Entwicklungshilfeausschusses (Development Assistance Committee, DAC) der OECD (Organization for Economic Cooperation and Development) regelmäßig die jeweilige Balance zwischen bi- und multilateraler EZ überprüft ${ }^{1}$, finden solche Untersuchungen Eingang in Allokationsentscheidungsprozesse von Gebern.

Zweitens ist das EZ-System durch einen längerfristigen Proliferationstrend der Akteure gekennzeichnet (Zimmermann und Smith 2011; Severino und Ray 2009). Dies betrifft unterschiedliche Akteurstypen wie private Akteure (v. a. Stiftungen), emerging economies als neue Geber (China, Indien, Mexiko etc.) und schließlich die Zunahme von multilateralen Finanzierungsmechanismen in der EZ. Die wachsende Zahl von Akteuren hat dazu beigetragen, das EZ-System als fragmentiert und nachteilig wahrzunehmen, wodurch u. a. Effizienzverluste aufgrund hoher Transaktionskosten entstehen. $80 \%$ aller Niedrigeinkommensländer (low-income countries, LICs) verzeichneten zwischen 2004 und 2009 eine Zunahme bei der Zahl der Geber, zum Teil nahm deren Zahl um mehr als $50 \%$ zu (OECD 2012a, S. 122). Für einige Beobachter (etwa Reisen 2009) ist die Zahl multilateraler Akteure ein Hauptfaktor, der zu Fragmentierung beigetragen hat. ${ }^{2}$ Andere Analysen erkennen hingegen eine gegenteilige Struktur, wonach die Konzentration multilateraler EZ insgesamt stärker sei und damit das multilaterale System weniger stark zu Fragmentierung beiträgt als bilaterale Geber. $^{3}$

\subsection{Unterscheidungen und Zwischenformen}

Die Unterscheidung zwischen bi- und multilateraler EZ ist eine der grundlegenden Unterscheidungen bei der Bereitstellung von EZ. Die Trennung deutet darauf hin, dass bestimmte EZ-Merkmale darauf zurückzuführen sind, ob es sich bei einem Geber um

111 von 23 DAC-Mitgliedsländern geben an, alle drei bis fünf Jahre die Balance zwischen biund multilateraler EZ zu überdenken (OECD 2012a, S. 30).

2 Reisen (2009) spricht daher von einem „Multilateral Donor Non-System“.

3 Siehe hierzu OECD (2012) sowie die näheren Erläuterungen weiter unten in diesem Beitrag. 
einen einzelnen Staat (bilaterale EZ) oder eine internationale Einrichtung (multilaterale EZ) handelt.

Die Kategorie multilaterale EZ geht von der Annahme aus, dass die einzelnen Geberbeiträge gepoolt und damit Teil der Leistung der bereitstellenden internationalen Einrichtung werden. ${ }^{4}$ In der Praxis ist diese Unterscheidung zwischen bi- und multilateraler EZ allerdings nicht immer eindeutig möglich, vielmehr gibt es verschiedene Zwischen- und Sonderformen (etwa so genannte multi-bi aid ${ }^{5}$ ), die EZ der Europäischen Union oder internationale Vereinbarungen, die zu sektoralen oder regionalen Quotierungen auch der bilateralen EZ führen (Klingebiel 2012). Beispielsweise werden multilateralen Stellen zunehmend Mittel mit einer bestimmten Zweckbedingung bereitgestellt, wodurch die Entscheidungsprozesse zur Verwendung der Mittel nicht mehr bei den entsprechenden Einrichtungen und ihren Aufsichtsgremien liegen. Umgekehrt werden zunehmend von internationalen Foren Vorgaben gemacht, für welche Zwecke die EZ auch bilateraler Geber eingesetzt werden soll, beispielsweise für bestimmte Ländergruppen oder spezifische Sektoren. Dies kann daher in umgekehrter Weise als ein Prozess interpretiert werden, der durch internationale Entscheidungen (vielfach nur mit empfehlendem Charakter) Einfluss auf bilaterale Allokationsentscheidungen nehmen soll.

\subsection{Umfang und Bedeutung ${ }^{6}$}

Gemessen an den Kernbeiträgen an multilaterale Einrichtungen (einschließlich an die EU) stellen DAC-Geber der OECD knapp 30\% ihrer EZ als multilaterale EZ zur Verfügung (2011: 27\% bzw. 37,6 Mrd. US-\$); dieser Anteil lag in früheren Jahren teilweise höher (2001: 33\%). Für die einzelnen DAC-Mitgliedsländer gibt es eine große Spannbreite bei der Frage, wie viel multilaterale EZ geleistet wird. Das weltweit größte EZGeberland, USA, stellt den mit Abstand geringsten Anteil für multilaterale Stellen bereit (2011: 11,5\%), Italien den höchsten (56,7\%); Deutschland befindet sich mit 34,4\% ,im oberen Mittelfeld““ (OECD 2013b). ${ }^{7}$

In den vergangenen Jahren haben solche Mittel stark zugenommen, die über multilaterale Kanäle bereitgestellt werden, aber eine Zweckbindung aufweisen, etwa hinsichtlich der Sektoren oder der Länder, für die diese Mittel eingesetzt werden müssen. Zum Teil lassen bilaterale Geber sehr spezifische - auch kleinere - Projekte über diesen Weg abwickeln. Diese Ressourcen sind daher keine „echten“ multilateralen Mittel, die als Kern-

4 Siehe hierzu die OECD-Definition zu multilateraler EZ. Demnach muss als eines von drei Merkmalen multilaterale EZ über folgendes Kennzeichen verfügen: ,pools contributions so that they lose their identity and become an integral part of its financial assets" (OECD 2011a, S. 23). Die beiden anderen Kriterien sind: 1) Die Aktivitäten der Einrichtung müssen zumindest teilweise darauf ausgerichtet sein, Entwicklung zu befördern, 2) die internationale Einrichtung hat Regierungen als Mitglieder oder ein Budget, das von dieser Institution selbstständig verwaltet wird.

5 Dabei handelt es sich um zweckbestimmte Mittel für bestimmte Aufgaben. Hierzu finden sich weiter unten nähere Erläuterungen.

6 Soweit nicht anders erwähnt stammen die Daten aus OECD 2011a.

7 Die Gründe hierfür werden weiter unten diskutiert. 


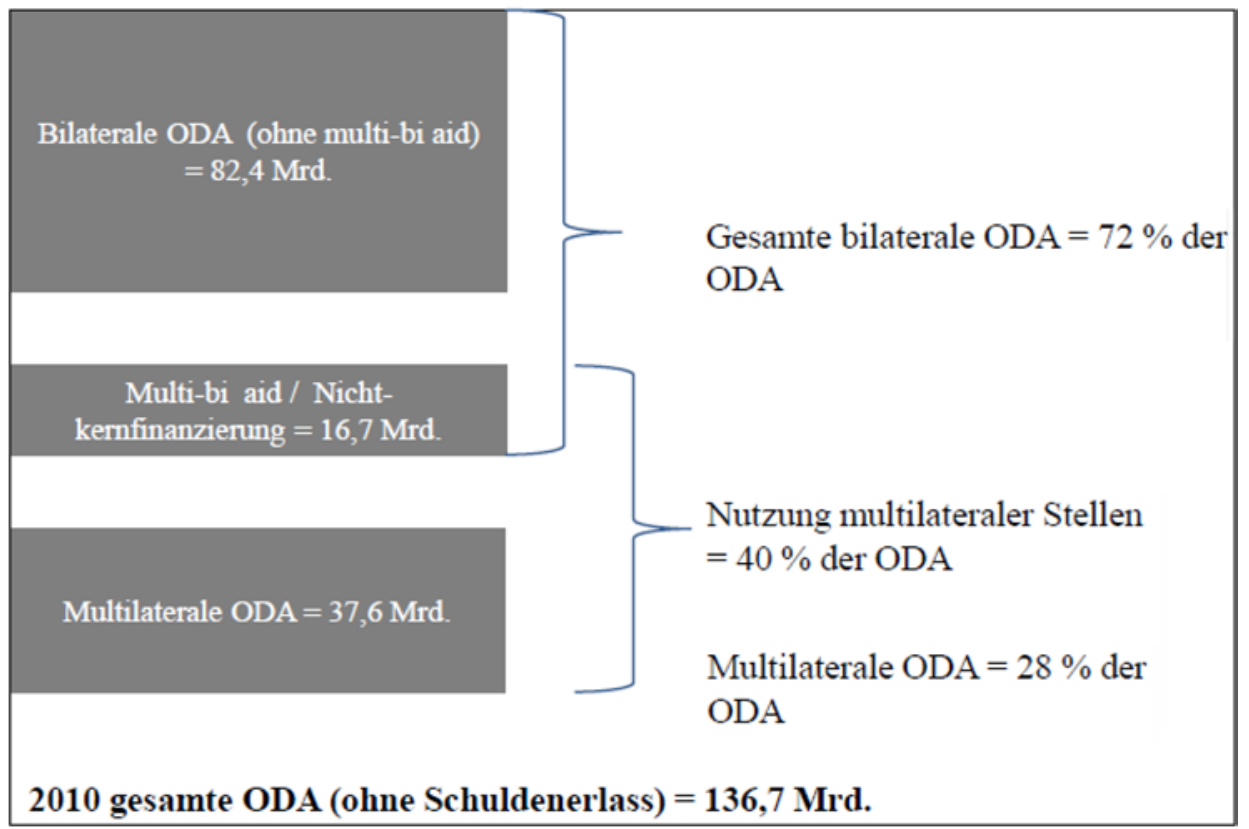

Abb. 1: Relevanz der bilateralen, multilateralen und multi-bi EZ (US-\$, 2010). (Quelle: OECD 2012b)

beiträge ${ }^{8}$ den internationalen Einrichtungen zur Verfügung stehen („Bilateralisierung der multilateralen EZ“), sondern werden über multilaterale Mechanismen verausgabt. Der Anteil dieser so genannten multi-bi EZ an der gesamten EZ beläuft sich derzeit auf $12 \%$ (2011: 16,5 Mrd. US-\$) und nimmt rasch zu (OECD 2013b). Einige Geber wie Australien, Kanada und die USA stellen über diesen Weg mehr Mittel als über Kernbeiträge zur Verfügung. Die Nachteile von multi-bi EZ sind erheblich und überwiegen mögliche Vorteile (Pilotierung bestimmter Ansätze etc.) bei weitem; dies gilt insbesondere dann, wenn es sich um Nicht-Kernbeiträge nur eines einzelnen Gebers handelt (also ein single trust fund), gegenüber einer Nicht-Kernfinanzierung durch mehrere Geber (multi-donor trust funds). Nicht-Kernbeiträge erhöhen beispielsweise die Transaktionskosten und untergraben die Governance-Strukturen multilateraler Institutionen und können so dazu beitragen, dass Mandate von Einrichtungen ,,verwässert“ werden, weil Anreize zur Übernahme verschiedenster Aufgaben gegeben werden (OECD 2012a, S. 47-49).

Das Verhalten ,neuer Geber“ gegenüber multilateraler EZ ist uneinheitlich. Die nicht dem DAC angehörenden EU-Mitgliedstaaten verfügen meist selbst nicht über Implementierungsstrukturen und stellen relativ hohe EZ-Anteile über die EU zur Verfügung. Russland und Brasilien nutzen insbesondere regionale Mechanismen, während China und Indien bilaterale Ansätze bevorzugen (OECD 2011a, S. 34; UNDESA 2010).

8 Also nicht an festgelegte Zwecke gebundene Mittel. 


\subsection{Akteure der multilateralen EZ}

Multilaterale EZ-Akteure sind heterogen etwa mit Blick auf ihre Bedeutung, Aufgabenstellung und regionale oder sektorale Ausrichtung. Hinsichtlich des zur Verfügung stehenden Volumens dominieren die EZ der EU-Institutionen sowie die Entwicklungsorganisation der Weltbank (International Development Association, IDA) und Regionalbanken (wie beispielsweise die Afrikanische Entwicklungsbank). Einige Akteure arbeiten weltweit, während andere auf eine bestimmte Region konzentriert sind. In Form des Global Fund zur Bekämpfung von HIV/AIDS, Tuberkulose und Malaria zählt ein so genannter vertikaler Fonds zu den wichtigsten multilateralen Akteuren. Außerdem besteht auf multilateraler Ebene zumindest in wichtigen Bereichen eine Aufgabenteilung zwischen Entwicklungsfinanzierung (Entwicklungsbanken) einerseits und Technischer Zusammenarbeit (UN-Fonds und -Programme) andererseits.

Auch wenn die Zahl der multilateralen EZ-Akteure mit rund 210 Organisationen, Fonds und Treuhandfonds ${ }^{9}$ groß ist, lässt sich eine Konzentration auf fünf Hauptakteure bzw. Akteursgruppen feststellen. Auf diese Akteure entfallen 81\% (Durchschnitt 2006-2010) aller Mittel, die von den DAC-Mitgliedern für multilaterale EZ aufgewendet werden (OECD 2013a, S. 12):

- Der Europäische Entwicklungsfonds (EEF) und das EZ-Budget der EU (36\%).

- Die zur Weltbankgruppe gehörende IDA (22\%).

- Die UN-Fonds und -Programme (9\%).

- Die Asiatische und die Afrikanische Entwicklungsbank (3 bzw. $5 \%$ ).

- Der Global Fund zur Bekämpfung von HIV/AIDS, Tuberkulose und Malaria (7\%).

Auf die übrigen multilateralen Stellen entfielen nur 19\% der gesamten multilateralen EZ.

\subsection{Die spezifische Rolle der EU}

Die EZ der EU-Institutionen, finanziert aus Mitteln des Europäischen Entwicklungsfonds und zu einem geringeren Anteil aus europäischen Haushaltsmitteln, beläuft sich auf rund $18 \%$ der gesamten EZ der EU-Mitgliedstaaten des DAC, womit die Europäische Kommission größter Geber in der EU ist (Gänzle et al. 2012; Klingebiel 2012). Die EZ der EU-Institutionen hatte 2010 einen Umfang 13 Mrd. US-\$; die im DAC vertretenen EUMitgliedstaaten stellen insgesamt mehr als die Hälfte (2010: 55 \%) der gesamten weltweiten EZ der DAC-Mitglieder zur Verfügung (OECD 2011b, S. 140, 154-155).

Die EU als supranationaler Geber ist ein spezifischer Fall, der nur sehr eingeschränkt der „multilateralen EZ“ zugeordnet werden kann. ${ }^{10}$ Die EU ist das einzige Mitglied des DAC, das in dreifacher Funktion in Erscheinung tritt. 1) Sie stellt zum einen selbst EZ für

$9 \mathrm{Mehr}$ Informationen hierzu bietet der Link http://www.oecd.org/dac/aid-architecture/12_10_02\%20Policy\%20Briefing\%20on\%20Multilateral\%20Aid_draft_final_draft $\% 20$ \%282\%29.pdf (Zugegriffen: 14. März 2013).

10 So heißt es im DAC-Bericht 2011: „Even though it is often presented as a multilateral in DAC publications, the EU is an individual donor with its own development policy and resources" (OECD 2011b, S. 155). 
Entwicklungsländer bereit. 2) Zum anderen tritt die EU auch als Geber gegenüber multilateralen Einrichtungen auf und leitet entsprechende Mittel weiter. 3) Zusätzlich wirkt die Union als Katalysator zwischen den EU-Mitgliedstaaten sowie der Kommission für abgestimmte Vorgehensweisen gegenüber multilateralen Einrichtungen und Partnerländern. Insgesamt wäre es angemessen, die EZ der EU nicht als Teil der multilateralen EZ zu subsumieren, sondern als kollektiven Bilateralismus zu verstehen (Klingebiel 1993). Eine solche neue Zuordnung wäre eventuell bei Geberländern relevant, die Obergrenzen für EZ-Beiträge an multilaterale Stellen festgelegt haben (OECD 2012a, S. 31).

Die Frage der Komplementarität im Bereich der EZ stellt sich für die EU in mehrfacher Hinsicht (Grimm 2010; Furness 2012). Vorrangig geht es um die Komplementarität zwischen Union und Mitgliedstaaten. Der Vertrag von Maastricht sah vor, dass die Politik der EU Institutionen die Politik der Mitgliedstaaten ergänzen soll. Die Bedeutung der EU als eigenständiger Geber wurde durch den Vertrag von Lissabon weiter gestärkt. Die Politik der EU und ihrer Mitgliedstaaten sollen sich nun gegenseitig ergänzen (shared parallel competences). Eine weitere wichtige und zunehmend diskutierte Dimension bezieht sich auf die Komplementarität der EZ zwischen den EU-Mitgliedstaaten.

Die Wirksamkeit der gemeinschaftlichen EZ der EU-Institutionen ist seit Ende der 1990er Jahre durch eine Reihe von Reformen deutlich verbessert worden (Grimm 2010). Weitere wichtige Herausforderungen für die EU und ihre Mitgliedstaaten bestehen bei der Koordinierung der europäischen EZ. Der Verhaltenskodex zur Arbeitsteilung von 2007 (division of labour) und die Initiativen zur Einführung gemeinsamer Programmierungsstrategien (joint programming) zielen hierauf ab.

\section{Multilaterale EZ: Stärken und Schwächen}

\subsection{Klassische Perspektiven auf die Stärken und Schwächen bi- und multilateraler EZ}

Die bilaterale EZ hat aus Sicht einzelner Geberländer verschiedene Vorteile. Der Geber kann sehr viel unmittelbarer die Ausrichtung der Hilfe bestimmen, etwa hinsichtlich der Themen und der Länderauswahl. Dies verschafft ihm eine höhere Sichtbarkeit sowohl im eigenen Land (gegenüber dem jeweiligen Parlament, der Öffentlichkeit etc.) als auch gegenüber dem Partnerland. Ebenfalls lassen sich etwaige Eigeninteressen sehr viel direkter mit bilateraler EZ verfolgen, etwa durch Formen der direkten oder indirekten Bevorzugung von Unternehmen aus dem Geberland oder durch die Instrumentalisierung von EZ zur außenpolitischen Sanktionierung von Partnerländern; direkte Eigeninteressen verringern meist den eigentlich angestrebten entwicklungspolitischen Nutzen von EZ. ${ }^{11}$ Bilaterale Geber nehmen zudem vielfach für sich in Anspruch, rascher, flexibler und unbürokratischer als multilaterale Akteure agieren zu können (Klingebiel 2012). Einige

11 So sind beispielsweise wegen des fehlenden internationalen Wettbewerbs liefergebundene Leistungen im Durchschnitt teurer und können qualitativ nachteilig gegenüber internationalen Ausschreibungen sein. Die Kosten durch Lieferbindung liegen rund 15 bis 30\%, im Fall von Nahrungsmittelhilfen sogar um rund $40 \%$ über denen ohne Lieferbindung (Clay et al. 2009, S. 1). 
Geber wie die Schweiz und Deutschland haben Obergrenzen für den Anteil von multilateraler EZ festgelegt (OECD 2012a, S. 31-32), um bilaterale EZ zu befördern und dadurch u. a. die Sichtbarkeit und Gestaltungsmöglichkeiten des Gebers zu stärken. Eine entwicklungspolitische Begründung im Sinne der Aid Effectiveness-Agenda für eine solche automatische Begrenzung oder feste Quotierung lässt sich nur schwerlich finden.

Multilaterale EZ bietet andere tatsächliche oder vermeintliche Vor- und Nachteile. Partnerregierungen verfügen teilweise über bessere Mitwirkungsmöglichkeiten an Entscheidungsprozessen von multilateraler EZ, weil sie selbst in den Aufsichtsgremien vertreten sind (etwa UN-Fonds und -Programme). Dies erhöht die Legitimität der EZ in den Partnerländern (Fues und Klingebiel 2007). Eigeninteressen von einzelnen Geberländern können sich deutlich weniger niederschlagen, multilaterale EZ gilt daher als weniger politisiert und interessengeleitet; die ownership von Partnerländern kann daher für multilaterale EZ ausgeprägter sein. Einzelne Forschungsarbeiten (Keohane et al. 2009) deuten darauf hin, dass multilaterale Institutionen und multilaterale EZ zudem dazu beitragen können, Demokratisierungsprozesse in den geförderten Ländern zu unterstützen, indem beispielsweise die Rechte von Minderheiten gestärkt werden.

Durch das Zusammenbringen von Ressourcen (pooling) können außerdem Skalenerträge erzielt und größere Aufgaben übernommen werden, die einzelne bilaterale Geber überfordern würden; dadurch kann das Risiko eines kleinteiligen Vorgehens („Projektitis“) reduziert werden. Außerdem können größere Risiken (etwa in fragilen Ländern) gemeinschaftlich besser als bilateral übernommen und das Risiko von unter- (aid orphans) oder überfinanzierten (aid darlings) Ländern verringert werden, da multilaterale Mechanismen eine gleichmäßigere Verteilung von EZ zwischen Ländern erreichen (OECD 2012a, S. 20). Multilaterale Akteure nehmen außerdem z. T. andere Aufgaben wahr als bilaterale Geber. Einige UN-Einrichtungen haben beispielsweise wichtige internationale Normensetzungsfunktionen, die von bilateralen Gebern nicht erbracht werden können (Fues und Klingebiel 2007; Stokke 2009; Thakur und Weiss 2009). Hinsichtlich von EU-Institutionen ist ein Kernargument, dass diese nicht nur einen weiteren europäischen Geber bilden, sondern in zunehmendem Maße eine Koordinierungsfunktion für das europäische EZ-System haben (Orbie 2012).

\subsection{Neuere Aspekte zu (multilateraler) EZ}

Neben den dargestellten Aspekten, die teilweise bereits in den 1960er und 1970er Jahren diskutiert wurden, sind weitere Aspekte zunehmend wichtig:

- EZ-Wirksamkeit und Perspektive der Partnerländer: Ob bi- oder multilaterale EZ bestimmte Vor- oder Nachteile aufweist, sollte vorrangig aus Sicht der Partnerseite und damit der Perspektive der „Wirksamkeit“ betrachtet und bewertet werden (Klingebiel 2012). Wenn ein fragmentiertes EZ-System insgesamt nachteilig für die Partnerseite ist (hohe Transaktionskosten, wenig Transparenz, hohe Overhead-Kosten zum Unterhalt des Systems etc.), stellt sich nicht vorranging die Frage nach „bi- und/ oder multilateraler EZ“ (wie dies in der Vergangenheit oft der Fall war), sondern grundsätzlicher nach einer sinnvollen Angebotsstruktur auf der Geberseite. Die Fokussierung auf „,bilateral“ oder „,multilateral“ ist daher nur begrenzt hilfreich; viel- 
mehr spielen andere Fragen (etwa die EZ-Modalitäten, Möglichkeiten zur Nutzung nationaler Systeme etc.) eine große Rolle.

- Multilaterale EZ bzw. eine weitere Multilateralisierung kann daher als ein Beitrag zur Umsetzung der EZ-Wirksamkeitsagenda (aid effectiveness) interpretiert werden (Manning 2012, S. 10). Die Bereitstellung von EZ über multilaterale Stellen führt zu Effizienzgewinnen (Abläufe werden rationalisiert, Mittel gebündelt etc.); gleichzeitig deuten die Forschungsergebnisse überwiegend auf den effektiven Einsatz von Mitteln über multilaterale Mechanismen hin. ${ }^{12}$

- „Neue Sichtbarkeit“: Vor dem Hintergrund neuer Vorgehensweisen auf Länderebene (größere Bedeutung von geberübergreifenden Sektordialogen etc.) und neueren EZ-Ansätzen zur Finanzierung von Programmen der Partnerseite (Korbbeiträge, Budgethilfen etc.) stellt sich die Frage der vermuteten besseren Sichtbarkeit bei der bilateralen EZ heute in veränderter Form. Partnerregierungen und in Partnerländern vertretene Geber beurteilen EZ-Beiträge deutlich stärker mit Blick auf die entwicklungspolitischen Wirkungen und die Integration in nationale Prozesse des Partnerlandes (Vollmer 2012) und weniger nach der Zurechnungsfähigkeit zu einem bestimmten Geber. Daher tritt das einzelne Projekt, das einem spezifischen Geber zuzuordnen ist, in den Hintergrund bzw. wird sogar als entwicklungspolitisch nachteilig betrachtet (Verzerrung der Rechenschaftslegungs-Strukturen). Eine hohe Sichtbarkeit kann z. B. in Partnerländern teilweise eher über Budgethilfen und andere Programmfinanzierungsbeiträge erreicht werden als mit klassischen vorzeigbaren bilateralen Projekten. Die Beteiligung an Dialogprozessen vor Ort hat einen zunehmend hohen Stellenwert für Sichtbarkeit. Insgesamt verliert dadurch das klassische Argument für bilaterale EZ - nämlich das der besseren Sichtbarkeit im Partnerland - an Bedeutung.

- Länder- und sektorbezogene Arbeitsteilung: Vor dem Hintergrund der Wirksamkeitsdebatten sind Fragen der Arbeitsteilung zwischen Gebern von großer Bedeutung. Die angestrebte Länderkonzentration von bilateralen Gebern führt u. a. dazu, dass in einer Reihe von Partnerländern (hierbei z. T. überproportional ,schwierige Partnerländer“) die Zahl der bilateralen Geber und das EZ-Volumen abnimmt, während zumindest die großen multilateralen EZ-Akteure ein breites Vertretungsnetz bewusst weiter unterhalten sollen (multilaterale EZ als donor of last resort). ${ }^{13}$ Ähnliches gilt für Fragen der sektoralen Arbeitsteilung innerhalb von Partnerländern.

- Globale öffentliche Güter (global public goods): Der Kontext, in dem Entwicklungszusammenarbeit stattfindet, verändert sich mit einem hohen Tempo. Entwicklungspolitische Herausforderungen wurden in der Vergangenheit insbesondere als Herausforderung auf Ebene des jeweiligen Staates betrachtet. Aufgrund der grenzüberschreitenden Dimension von zahlreichen Themen (Sicherheit, Auswirkungen des Klimawandels, Migration, Nahrungsmittelsicherheit etc.), ist eine solche Sichtweise kaum noch angemessen. Entwicklungspolitische Aufgaben werden zunehmend im Zusammenhang mit der Bereitstellung von öffentlichen Gütern behandelt (Kaul et al. 2003; Kaul 2013).

12 Siehe hierzu ausführlicher das folgende Kapitel.

13 So arbeitet das Department for International Development (DFID) nur noch mit 28 Ländern zusammen (DFID 2012, S. 8), während die EU in rund 150 Ländern aktiv ist (Parlament UK 2012). 
Um an der Bereitstellung globaler und regionaler öffentlicher Güter mitzuwirken, haben multilaterale Akteure Vorzüge. Die Schwierigkeiten, die bei kollektiven Handlungsmustern entstehen, dürften durch multilaterale Akteure besser zu bearbeiten sein als durch eine Vielzahl von einzelstaatlichen Ansätzen.

- Normen und Standards: Schließlich haben u. a. die Beispiele der Millenniumsentwicklungsziele und die EZ-Wirksamkeitsagenda ${ }^{14}$ bestätigt, dass die Setzung von internationalen Normen und Standards für globale, regionale und nationale Politiken (der Partnerländer, der Geber etc.) nur durch multilaterale Akteure erfolgen kann. Aufgrund ihrer universellen Legitimität sind hierzu nur multilaterale Organisationen in der Lage.

\section{Wirksamkeit der multilateralen EZ}

Es lassen sich vor allem zwei Bewertungsmaßstäbe für multilaterale EZ nutzen. Dies sind zum einen gezielte Leistungsanalysen zum multilateralen EZ-System bzw. zu einzelnen multilateralen Institutionen. Zum anderen sind dies Indikatoren, die von der OECD in den vergangenen Jahren eingeführt wurden und Hinweise zur Funktionsfähigkeit multilateraler EZ geben.

\subsection{Leistungsanalysen}

In den letzten fünf bis zehn Jahren sind verstärkt Bemühungen unternommen worden, die Leistungsfähigkeit von Gebern - teilweise spezifisch für die multilateralen Institutionen - systematischer zu bewerten. Zu den wichtigsten Analysen zählen:

- Die Evaluierung der Paris-Erklärung, bei der es um die Überprüfung der Indikatoren zur EZ-Wirksamkeit geht. Die umfassendste Untersuchung von Wood et al. (2011) bezieht allerdings nicht die EZ der EU und die IDA ein.

- Das von 16 Gebern getragene und seit 2002 bestehende Netzwerk zur gemeinschaftlichen Bewertung der multilateralen EZ-Wirksamkeit (Multilateral Organisation Performance Assessment Network, MOPAN ${ }^{15}$ ) durch Geber. Pro Jahr nimmt MOPAN vier bis sechs gemeinsame Analysen vor (OECD 2013a).

- Das Quality of Official Development Assistance Assessment (QuODA) (Birdsall und Kharas 2010), das insgesamt 31 bi- und multilaterale Geber einbezieht.

- Geber-Studien und Untersuchungen zum multilateralen System wie der 2011 von DFID durchgeführte Multilateral Aid Review; ähnliche Untersuchungen wurden von Australien, den Niederlanden und Schweden vorgenommen (OECD 2013a, S. 22; OECD 2013b). In Deutschland hat das Bundesministerium für wirtschaftliche Zusammenarbeit und Entwicklung (BMZ 2013) drei Kriterien zur systematischen Bewertung multilateraler Akteure entwickelt: 1) Entwicklungspolitische Relevanz/

14 Daher sind Bemühungen darauf gerichtet, die internationalen Diskussionen über EZ-Wirksamkeit stärker unter Einbeziehung der UN zu organisieren.

15 Ausführliche Informationen hierzu bietet der Link http://www.mopanonline.org/ (Zugegriffen: 11. Okt. 2013). 
Mandat einer Organisation, 2) Leistungsfähigkeit einer Organisation und 3) Einflussmöglichkeiten Deutschlands. Im Gegensatz zu MOPAN unterscheiden sich die Analysen einzelner Geber, da die jeweiligen Geberpräferenzen das Untersuchungsprofil stark prägen (Prioritäten des Gebers, die möglichweise in der Form nicht Teil des Profils der Institution sind), die nicht der Rationalität und Ausrichtung der multilateralen Einrichtung entsprechen müssen.

- Auf einzelne Akteure bezogene Berichte. ${ }^{16}$

Verallgemeinernde Schlussfolgerungen zur Qualität der bilateralen EZ einerseits und der multilateralen EZ andererseits sind trotz der vorliegenden Untersuchungen nur beschränkt möglich. Dies liegt an den sehr heterogenen Gebergruppen (sowohl auf Seiten der bi- als auch der multilateralen Akteure) sowie der sehr unterschiedlichen Untersuchungsmethoden und Herangehensweisen.

Dennoch deuten die meisten Untersuchungsergebnisse insgesamt darauf hin, dass multilaterale EZ durchschnittlich gute Wertungen erhält. QuODA zeigt beispielsweise auf, dass im Durchschnitt multilaterale EZ von vier erfassten Qualitätsdimensionen in drei Dimensionen besser als bilaterale Geber abschneidet (Birdsall und Kharas 2010, S. 24-25). In der Tendenz unterstützen andere Studien diese Einschätzungen (Picciotto 2011).

In welchem Umfang und in welcher Weise Untersuchungen zur Leistungsfähigkeit multilateraler EZ tatsächlich Geberallokationsentscheidungen beeinflussen, lässt sich bislang nur unzureichend einschätzen. Befragungen von DAC-Gebern legen den Schluss nahe, dass in der Eigendarstellung entsprechende Analysen zu den wichtigen Grundlagen für Allokationsentscheidungen zählen. ${ }^{17}$

In der Perzeption der EU-Bürger wird eine gute Qualität in der Zusammenarbeit von internationalen Organisationen im Vergleich zu bilateralen Programmen geteilt. Demnach schätzen in 26 von 27 Ländern die Bürger die Qualität von großen internationalen Organisationen besser als die der bilateralen Ansätze ein (OECD 2012a, S. 30). Mit Blick auf die USA zeigen Forschungsergebnisse (Milner und Tingley 2012) gleichzeitig, dass trotz vorhandener Erkenntnisse zur Wirksamkeit multilateraler EZ die öffentliche Unterstützung hierfür gering ist. Dies wird anhand des Principal-Agent-Modells mit dem grundsätzlichen Zielkonflikt zwischen einem effizienten und effektiven Mitteleinsatz (etwa zugunsten des agent Weltbank) und dem Steuerungsverlust durch den principal (in dem Fall den USA) erklärt. Das heißt, dass trotz möglicher Kompetenzvermutungen des multilateralen Systems aufgrund der geringeren Steuerungsmöglichkeiten ein multilaterales Vorgehen ggf. nur geringe Unterstützung erfährt.

\subsection{Leistungsindikatoren}

In der Vergangenheit wurden zur Einschätzung von Gebern bereits eine Vielzahl von (überwiegend) Input-Indikatoren erhoben und in der Forschung verwendet. Hierzu zählen der Gesamtumfang von EZ (Official Development Assistance, ODA), regionale, ein-

16 So etwa der Bericht von Bigsten et al. (2011) zur EU.

17 Siehe hierzu die Nennungen einer DAC-Geberbefragung zur multilateralen EZ (OECD 2012a, S. 32). 
kommensbezogene und sektorale Verteilungsindikatoren, Verteilung auf Instrumente und Vergabeverfahren (Anteil von Humanitärer Hilfe etc.; liefergebundene EZ).

Ergänzende neuere Daten der OECD betreffen folgende quantitative und qualitative Aspekte:

1. Country Programmable Aid (CPA) trägt der Kritik Rechnung, dass viele als ODA ausgewiesene Leistungen nur begrenzt durch die Partnerländer steuerbare Beiträge darstellen. Leistungen wie „Studienplatzkosten“ in den Geberländern für Entwicklungsländerangehörige etc. sind nicht oder nur gering durch die Empfängerländer „programmierbar“. CPA ist eine Unterkategorie von ODA, die bestimmte ODA-fähige Ausgaben (etwa Administrationskosten) der Geber nicht umfasst und daher einen besseren Anhaltspunkt zum möglichen Nutzen der EZ aus Partnerlandsicht bietet (OECD 2013c). Die CPA-Werte für multilaterale Institutionen sowie die EU-Institutionen liegt dabei im Durchschnitt deutlich höher als bei den bilateralen EZ der DAC-Geber. Für 2010 lag der CPA-Anteil für alle multilateralen Stellen (ohne EU) bei 74,0\%, für die EU-Institutionen bei 72,5\% und für die bilaterale EZ der DAC-Geber bei 55,1\%. ${ }^{18}$

2. Die OECD hat in den vergangenen Jahren quantitative Analysen vorgelegt, die die „Signifikanz“ von EZ-Beziehungen (significance of an aid relation), die EZ-Konzentrationsrate (concentration ratio) ${ }^{19}$ und die Fragmentierungsrate (fragmentation ratio $)^{20}$ erfassen (OECD 2011c, 2012a). Eine Schätzung der Leistungsfähigkeit von Gebern vor dem Hintergrund dieser Daten ist aus verschiedenen Gründen schwierig. ${ }^{21}$ Gleichwohl deuten die verfügbaren Daten darauf hin, dass Fragmentierungsphänomene relativ stärker durch bilaterale als durch multilaterale EZ hervorgerufen werden. Die EZ-Konzentrationsrate der Geber ${ }^{22}$ liegt demnach bei der multilateralen EZ (einschließlich EU) bei $65 \%$ (ohne EU: $62 \%$ ), bei der bilateralen EZ der DAC-Länder hingegen bei 54\% (OECD 2013a, S. 22; Tab. 1).

\section{Schlussfolgerungen}

Grundsätzlich gab es in der Vergangenheit zwei implizite Sichtweisen auf das multilaterale EZ-System: Eine Perspektive war geprägt von der Prämisse eines funktionsfähigen „Patchwork-Ansatzes“ für globale Herausforderungen. Auch wenn es zahlreiche indivi-

18 Berechnet nach Daten aus OECD (2013b).

19 Aus Gebersicht der Grad von ,signifikanten“ Beziehungen zu Partnerländern.

20 Aus Partnerländersicht „signifikante“ Geberbeziehungen.

$21 \mathrm{Ob}$ eine finanziell signifikante bzw. nicht-signifikante EZ-Beziehung für das Partnerland sinnvoll ist, hängt auch von verschiedenen weiteren Faktoren ab, die möglicherweise sogar wichtiger sein können (etwa möglicherweise geringe Transaktionskosten, wenn es sich um kleinere Finanzierungsbeiträge zu stark harmonisierten Programmen handelt). Bei der Zahl der Partnerländer multilateraler Institutionen ist etwa von hoher Bedeutung, ob das Mandat eine umfassende oder eine regionale Zuständigkeit vorsieht (etwa das universelle Vorgehen der UN-Fonds und -Programme gegenüber den Regionalbanken).

22 Die EZ-Konzentrationsrate misst das Verhältnis von „signifikanten“ zu „,nicht-signifikanten“ EZ-Beziehungen eines Gebers. 
Tab. 1: Profil und Leistungen multilateraler Institutionen (Kernbeiträge 2010). (Quelle: OECD 2013b)

\begin{tabular}{|c|c|c|c|c|c|}
\hline & $\begin{array}{l}\mathrm{CPA}+{ }^{\mathrm{a}} \\
(\mathrm{mio} . \mathrm{USD})\end{array}$ & \begin{tabular}{|l|}
$\begin{array}{l}\text { Share of Global } \\
\mathrm{CPA}^{\mathrm{a}} \text { (in \%) }\end{array}$ \\
\end{tabular} & $\begin{array}{l}\text { No. of } \\
\text { relations }\end{array}$ & $\begin{array}{l}\text { No. of signifi- } \\
\text { cant relations }\end{array}$ & $\begin{array}{l}\text { Concentration } \\
\text { ratio (in \%) }\end{array}$ \\
\hline Total multilaterals & 34.360 & 35,7 & 1.594 & 1.030 & 65 \\
\hline $\begin{array}{l}\text { Total multilaterals } \\
\text { excl. EU }\end{array}$ & 24.485 & 25,5 & 1.445 & 902 & 62 \\
\hline EU institutions ${ }^{\mathrm{b}}$ & 9.875 & 10,3 & 149 & 128 & 86 \\
\hline Global Fund & 2.997 & 3,1 & 113 & 73 & 65 \\
\hline IDA & 10.074 & 10,5 & 78 & 70 & 90 \\
\hline $\begin{array}{l}\text { IMF (Concessional } \\
\text { Trust Fund) }\end{array}$ & 1.346 & 1,4 & 37 & 29 & 78 \\
\hline $\begin{array}{l}\text { Regional Development } \\
\text { Bank Funds }\end{array}$ & 4.174 & 4,3 & 104 & 94 & 90 \\
\hline AfDF & 1.515 & 1,6 & 38 & 31 & 82 \\
\hline AsDF & 1.927 & 2,0 & 27 & 25 & 93 \\
\hline CarDB & 69 & 0,1 & 14 & 14 & 100 \\
\hline IDB & 662 & 0,7 & 25 & 24 & 96 \\
\hline $\begin{array}{l}\text { UN Funds and } \\
\text { Programmes } \\
\end{array}$ & 2.438 & 2,5 & 534 & 288 & 54 \\
\hline UNDP & 515 & 0,5 & 136 & 75 & 55 \\
\hline UNFPA & 282 & 0,3 & 117 & 75 & 64 \\
\hline UNHCR & 187 & 0,2 & 86 & 43 & 50 \\
\hline UNICEF & 768 & 0,8 & 120 & 51 & 43 \\
\hline UNRWA & 452 & 0,5 & 4 & 4 & 100 \\
\hline WFP & 235 & 0,2 & 71 & 40 & 56 \\
\hline Other UN & 615 & 0,6 & 298 & 201 & 67 \\
\hline IAEA & 51 & 0,05 & 98 & 67 & 68 \\
\hline IFAD & 462 & 0,48 & 79 & 53 & 67 \\
\hline UNAIDS & 51 & 0,05 & 103 & 65 & 63 \\
\hline $\begin{array}{l}\text { UN Peacebuilding } \\
\text { Fund }\end{array}$ & 51 & 0,05 & 18 & 16 & 89 \\
\hline Total DAC countries & 61.764 & 64,3 & 1.719 & 933 & 54 \\
\hline $\begin{array}{l}\text { Total DAC members } \\
\text { incl. EU }\end{array}$ & 71.639 & 74,5 & 1.868 & 1.061 & 57 \\
\hline
\end{tabular}

duelle Beispiele für ein wenig kohärentes multilaterales System gebe, müsse dies nicht zwangsläufig bedeuten, dass angesichts der Vielzahl unterschiedlicher globaler, regionaler und nationaler Herausforderungen kein synergetisches Potenzial durch die verschiedenen multilateralen Akteure erzeugt wird. Die Vielzahl von Aufgaben und Problemstellungen bedingt vielmehr unterschiedliche Akteure und dadurch uneinheitliche Lösungsansätze. 
Eine zweite Perspektive geht hingegen davon aus, dass das multilaterale System durch Wirkungsverluste und von Fragmentierung gekennzeichnet ist. Das System ist demnach geprägt durch eine große Akteursvielfalt, die oftmals überlappende Aufgaben in einer wenig koordinierten Weise übernehmen und häufig nur über eine geringe Mittelausstattung verfügen. Dies führe im Ergebnis für die Partnerländer zu Effizienz- und Effektivitätsverlusten (Transaktionskosten, Wirkungsverluste etc.) in der Bereitstellung von EZ.

Tatsächlich ist eine Proliferation von Akteuren Kennzeichen des gesamten EZ-Systems. Die multilateralen EZ-Mechanismen sind ein Bestandteil des Systems und tragen dazu bei, dass Wirkungs- und Effizienzverluste entstehen, selbst wenn die bilateralen Geber in einem größeren Umfang Verantwortung hierfür tragen. Sinnvoll ist es daher, nicht isoliert multilaterale Institutionen in den Blick zu nehmen, sondern unter Einbeziehung aller Geberakteure.

Eine Reihe von Untersuchungen ${ }^{23}$ der letzten Jahre hat zu einem besseren Verständnis des multilateralen EZ-Systems und seiner Akteure beigetragen. Die Analysen haben deutlich gemacht, dass die multilaterale EZ im Durchschnitt positiv zu bewerten ist, auch wenn es zwischen den Institutionen teilweise ein erhebliches Leistungsgefälle gibt. Untersuchungen stützen nicht die These, dass die Qualität multilateraler EZ unzureichend ist. Darauf aufbauend sollte eine Forschungsfragestellung für die Zukunft sein: Wie wirken sich diese Untersuchungen auf Allokationsentscheidungen der Geber aus? Tragen sie zu veränderten Präferenzen und Entscheidungen von Geberregierungen und -Parlamenten bei? Oder unterliegen Allokationsentscheidungen stärker anderen Kriterien?

Die vergangenen Jahre haben einen Trend in Richtung Nutzung des multilateralen Systems für spezifische Geberinteressen deutlich werden lassen (Mavrotas und Villanger 2006). Erkennbar wird dies durch das veränderte Finanzierungsverhalten, bei dem Ziele einzelner Geber festgelegt und durch multilaterale Institutionen durch multi-bi EZ umgesetzt werden. Den Trend verstärken Geberländer ebenfalls durch in Auftrag gegebene Leistungsanalysen, die dem Zielsystem des jeweiligen Gebers und nicht dem Zielsystem der jeweiligen multilateralen Einrichtung verbunden sind. Dadurch werden gerade die mit multilateralen Gebern verbundenen Vorzüge vermindert und dadurch der Nutzen verringert. Der Trend bestätigt damit die Schwierigkeit, das bisherige EZ-System in ein umfassenderes System von global public policies zu überführen (Severino 2013).

Für die Allokationsdebatten (bilaterale und multilaterale Prioritäten) europäischer Geber ist die Frage der Zuordnung der EZ der EU von großer Bedeutung. Insgesamt lässt sich feststellen, dass sich die EZ der europäischen Institutionen deutlich unterscheidet von der Arbeit multilateraler Akteure. Die EU ist ein politischer Zusammenschluss, der als eigenständiger Akteur bei multilateralen Einrichtungen (etwa den Vereinten Nationen) in Erscheinung tritt. Die Gestaltungsmöglichkeiten einzelner Mitgliedstaaten sind deutlich ausgeprägter als bei multilateralen Einrichtungen mit strukturell anderen Governance-Gremien. Diesem Unterschied zwischen einem multilateralen Vorgehen und Ansätzen der EU sollte in der EZ (z. B. bei der Darstellung der bi- und multilateralen EZ) Rechnung getragen werden.

23 Siehe das vorangegangene Kapitel. 
Open Access Dieser Artikel unterliegt den Bedingungen der Creative Commons Attribution Noncommercial License. Dadurch sind die nichtkommerzielle Nutzung, Verteilung und Reproduktion erlaubt, sofern der/die Originalautor/en und die Quelle angegeben sind.

\section{Literatur}

Bigsten, A. L., Platteau, J. P., \& Tengstam, S. (2011). The aid effectiveness agenda. The benefits of going ahead. Brüssel: EU.

Birdsall, N., \& Kharas, H. (2010). Quality of official development assistance assessment. Washington, DC: Brookings Institution and the Center for Global Development.

BMZ - Bundesministerium für wirtschaftliche Zusammenarbeit und Entwicklung. (2013). Eckpunktepapier für die multilaterale Entwicklungspolitik. Bonn: BMZ.

Clay, E. J., Geddes, M., \& Natali, L. (2009). Untying aid: Is it working? An evaluation of the implementation of the Paris Declaration and of the 2001 DAC recommendation of untying ODA to the LDCs. Kopenhagen: Danish Institute for International Studies.

DFID - Department for International Development. (2011). Multilateral aid review. London.

DFID - Department for International Development. (2012). Annual report and account 2011-2012. London.

Fues, T., \& Klingebiel, S. (2007). Multilaterale Entwicklungszusammenarbeit. Die Rolle der Vereinten Nationen. In H. Volger (Hrsg.), Grundlagen und Strukturen der Vereinten Nationen (S. 219-241). München: Oldenbourg Verlag.

Furness, M. (2012). The Lisbon treaty, the European external action service and the reshaping of EU development policy. In S. Gänzle, S. Grimm \& D. Makhan (Hrsg.), The European Union and global development. An ,enlightened superpower" in the making? (S. 74-93). Houndmills: Palgrave Macmillan.

Gänzle, S., Grimm, S., \& Makhan, D. (Hrsg.). (2012). The European Union and global development. An ,enlightened superpower" in the making? Houndmills: Palgrave Macmillan.

Grimm, S. (2010). Zur Wirksamkeit europäischer Entwicklungspolitik. In J. Faust \& S. Neubert (Hrsg.), Wirksamere Entwicklungspolitik. Befunde, Reformen, Instrumente (S. 381-402). Baden-Baden: Nomos.

Kaul, I., Conceicao, P., Le Goulven, K., \& Mendoza, R. U. (Hrsg.). (2003). Providing global public goods: managing globalization. New York: Oxford University Press.

Kaul, I. (2013). Global public goods. A concept for framing the Post-2015 Agenda? DIE Discussion Paper 2/2013. Bonn: Deutsches Institut für Entwicklungspolitik/German Development Institute.

Keohane, R. O., Macedo, S., \& Moravcsik, A. (2009). Democracy-enhancing multilateralism. International Organization, 63(1), 1-31.

Klingebiel, S. (1993). Multilaterale Entwicklungspolitik. Aus Politik und Zeitgeschichte, Beilage zur Wochenzeitung Das Parlament, Bd. 12-13/93, 19. März 1993.

Klingebiel, S. (2012). Stellungnahme zur Öffentlichen Anhörung des Deutschen Bundestages. Ausschuss für wirtschaftliche Zusammenarbeit und Entwicklung (AwZ) zum Thema „Bilaterale und multilaterale Entwicklungszusammenarbeit“, 23. März 2012, Bonn.

Mahn, T. (2012). The financing of development cooperation at the United Nations. Why more means less. DIE Briefing Paper 8/2012. Bonn: Deutsches Institut für Entwicklungspolitik/ German Development Institute.

Manning, R. (2012). Aid as a second-best solution: Seven problems of effectiveness and how to tackle them. Working Paper No 2012/24. Helsinki: UNU-Wider.

Mavrotas, G., \& Villanger, E. (2006). Multilateral aid agencies and strategic donor behaviour. Discussion Paper No. 2006/02. Helsinki: UNU-Wider. 
Milner, H. V., \& Tingley, D. (2012). The choice of multilateralism. Foreign aid and American foreign policy. The Review of International Organizations, 8(3), 313-341.

North-South Institute. (2011). Canadian development report 2011. Global challenges. Multilateral solutions. Ottawa.

OECD. (2011a). 2011 DAC report on multilateral aid. Paris: OECD.

OECD. (2011b). Development co-operation report 2011. 50th anniversary edition. Paris: OECD.

OECD. (2011c). 2011 OECD report on division of labour. Addressing cross-country fragmentation of aid. Paris: OECD.

OECD. (2012a). The architecture of development assistance. Paris: OECD.

OECD. (2012b). Aid architecture. http://www.oecd.org/dac/aid-architecture/multilateralaid.htm. Zugegriffen: 14. Juni 2013.

OECD. (2013a). 2012 DAC report on multilateral aid. Paris: OECD.

OECD. (2013b). Country programmable aid. DAC countries. http://webnet.oecd.org/dcdgraphs/ CPA_donor/. Zugegriffen: 14. März 2013.

OECD. (2013c). Getting closer to the core - measuring country programmable aid. http://www. oecd.org/dac/stats/gettingclosertothecore-measuringcountryprogrammableaid.htm. Zugegriffen: 13. März 2013.

Orbie, J. (2012). The EU's role in development. A full-fledged development actor or eclipsed by superpower temptations? In S. Gänzle, S. Grimm \& D. Makhan (Hrsg.), The European Union and global development. An ,enlightened superpower" in the making? (S. 17-36). Houndmills: Palgrave Macmillan.

Parlament UK. (2012). EU aid instruments and organisations. http://www.publications.parliament. $\mathrm{uk} / \mathrm{pa} / \mathrm{cm} 201012 / \mathrm{cmselect} / \mathrm{cmintdev} / 1680 / 168005 . h t m$. Zugegriffen: 13. März 2013.

Picciotto, R. (2011). Multilateral development cooperation and the Paris process. The road to Busan. In North-South Institute (Hrsg.), Canadian development report 2011. Global challenges. Multilateral solutions (S. 55-76). Ottawa.

Reisen, H. (2009). The multilateral donor non-system. Towards accountability and efficient role assignment. Economics, Discussion Paper, Nr. 2009-18.

Severino, J.-M., \& Ray, O. (2009). The end of ODA. Death and rebirth of a global public policy. Working Paper Number 167. Washington, DC: Center for Global Development.

Severino, J.-M. (2013). La belle et la bête - Transmutations et reformulations dans les politiques globales. Hintergrundpapier für den European report on development 2013, Brüssel. http:// www.erd-report.eu/erd/report 2012/documents/bp/bgpapers/SeverinofinalFR.pdf. Zugegriffen: 14. Juni 2013.

Stokke, O. (2009). The UN and development: From aid to cooperation. Bloomington: Indiana University Press.

Thakur, R., \& Weiss, T. G. (2009). The UN and global governance: An unfinished journey. Bloomington: Indiana University Press.

UNDESA - Department of Economic and Social Affairs. (2010). International development cooperation report. Development cooperation for the MDGs. Maximising results. UNDocument. ST/ ESA/326. New York: UN.

Vollmer, F. (2012). Increasing the visibility and effectiveness of development cooperation. How to reconcile two competing objectives? Studies 67. Bonn: Deutsches Institut für Entwicklungspolitik/German Development Institute.

Wood, B., Betts, J., Etta, F., Gayfer, J., Kabell, D., Ngwira, N., Sagasti, F., \& Samaranayake, M. (2011). The evaluation of the Paris declaration. Phase 2. Final report. Aarhus: Danish Institute for International Studies.

Zimmermann, F., \& Smith, K. (2011). More actors, more money, more ideas for international development co-operation. Journal of International Development, 23(5), 722-738. 